\title{
Liens entre salariat et activité agricole : itinéraires professionnels de salariés d'élevage*
}

\author{
Sophie Madelrieux ${ }^{1}$ \\ Lucie Dupré2 \\ Nathalie Hostiou ${ }^{3}$ \\ Tienne Barbosa ${ }^{4}$ \\ Amaury Burlamaqui Bendahan ${ }^{5}$ \\ Jean-François Tourrand ${ }^{6}$ \\ ${ }^{1}$ Cemagref \\ UR DTM \\ Domaine universitaire \\ 2, rue de la Papeterie \\ BP 76 \\ 38402 St Martin d'Hères \\ France \\ <sophie.madelrieux@cemagref.fr> \\ 2 Inra \\ Mona/SAE2 \\ 65, avenue de Brandebourg \\ 94250 Ivry-sur-Seine \\ France \\ <dupre@ivry.inra.fr> \\ 3 Inra \\ Unité mixte de recherche Métafort \\ 63122 Saint Genès \\ Champanelle \\ France \\ <nhostiou@clermont.inra.fr> \\ ${ }^{4}$ Embrapa Amazonia Oriental \\ Trav. Dr. Enéas Pinheiro \\ CEP 66095-100 Caixa Postal \\ 48 Belém \\ Brésil \\ <tiennejo@yahoo.com.br> \\ ${ }^{5}$ Embrapa Roraima \\ Rodovia BR-174 \\ Km 8 Distrito industrial \\ 69301-970 Boa Vista \\ Brésil \\ <amaury@cpafrr.embrapa.br> \\ ${ }^{6}$ Cirad
}

Direction de la recherche et de la stratégie Montpellier

F-34398 France

<jean-francois.tourrand@cirad.fr>

\begin{abstract}
Résumé
Le salariat agricole se développe et les exploitations agricoles y ont de plus en plus recours, notamment dans des systèmes d'élevage herbivore traditionnellement familiaux. En élevage herbivore, peu de travaux s'y sont intéressés alors qu'il permet de repenser le fonctionnement des exploitations et d'assurer dans certains cas leur reproduction. Nous proposons ici de nous intéresser au salariat, du point de vue des salariés, et de caractériser une diversité d'itinéraires individuels menant au salariat agricole. Nos résultats permettent de révéler une diversité d'enchaînements de statuts professionnels dans l'exercice de l'élevage, qui peut conduire de salarié agricole à chef d'exploitation. Et par là même se dessinent des formes de liens entre activité agricole et salariat autres que l'emploi ou les fonctions affectées aux salariés dans les exploitations.
\end{abstract}

Mots clés : changement structurel ; ferme d'élevage ; main-d'œuvre ; mobilité de la main-d'œuvre.

Thèmes : économie et développement rural ; productions animales.

\section{Abstract \\ Relationship between wage labour and agricultural activity: Occupational paths of salaried employees in livestock farming}

Wage labour in farming is increasing and herbivorous livestock farming systems that were hitherto family-based are increasingly resorting to it. Few studies have been carried out on wage labour in this type of systems. Yet it impacts both the operation and reproduction of livestock farms. The issue we address here is that of wage labour from the salaried employee's point of view. We propose to characterize a diversity of individual paths that have led to wage labour. This study reveals a diversity of occupational status sequences, which can lead from a status of salaried employee to that of farmer. It thus reveals forms of relationships between wage labour and agricultural activity other than employment or the functions of a salaried employee on farms.

Key words: labour mobility; livestock farms; manpower; structural change.

Subjects: animal productions; economy and rural development.
C ontrairement à ce que pourraient laisser penser ces termes, agriculture familiale et salariat agricole ne sont pas deux réalités disjointes (Cournut et al., 2010 ; Dupré, 2010). Le développement du salariat agricole dans les agricultures familiales dans différentes régions du monde est patent d'après les statisti- ques de l'International Labour Organization (ILO, 2008), et il est un révélateur des mutations de l'agriculture - et plus particulièrement dans ce texte - de l'élevage familial. Le développement du salariat se justifie en France par l'accroissement des dimensions des exploitations et la diminution de la main-d'œuvre familiale:

\footnotetext{
*Pour citer cet article: Madelrieux S, Dupré L, Hostiou N, Barbosa T, Burlamaqui Bendahan A, Tourrand JF. Liens entre salariat et activité agricole: itinéraires professionnels de salariés d'élevage. Cah Agric 2010 ; 19 : 354-8. doi : 10.1684/agr.2010.0426.
} 
embaucher un salarié seul, en groupement d'employeurs ou dans le cadre de coopératives d'utilisation du matériel agricole (CUMA) (Harff et Lamarche, 1998) est désormais une option d'organisation du travail reconnue d'avenir. Dans un contexte très différent, au Sénégal, le développement récent du salariat spécialisé dans le gardiennage et la transhumance du bétail dans les campements peuls traduit une évolution du système d'activités des familles rurales et le moindre intérêt des cadets qui, par leur rang familial, étaient destinés traditionnellement à cette fonction (Wane et al., 2010). Dans d'autres pays, en Amérique du Sud ou au Vietnam, les journaliers ou les tâcherons affectés à l'entretien des clôtures ou des surfaces fourragères (Hostiou et Dedieu, 2009), mais aussi les vachers permanents font partie du paysage de l'emploi agricole et rural. Ils contribuent au travail dans différents types d'exploitations, dont les exploitations familiales (Neiman, 2001 ; Hostiou et al., 2010).

Les interactions entre agriculture familiale et salariat sont souvent abordées du point de vue de l'exploitation agricole et de l'employeur (fonctions du salarié et relations à l'employeur) ou par rapport à l'activité agricole en général (l'emploi), mais sont rarement considérées du point de vue du salarié lui-même. En effet, dans la littérature, ces liens sont caractérisés par les tâches réalisées par les salariés selon leur rythme de présence (Chabanet et al., 2000) ou par leur fonction dans l'exploitation : salarié "associé ", second d'exploitation, technicien spécialisé, commis, manœuvre (Cedag, 2001). D'autres abordent ces liens par rapport à l'emploi agricole et la mobilité des salariés (Findeis et al., 2002). Mais qui sont les salariés agricoles, d'où viennent-ils, quel futur envisagent-ils ? À partir d'un ensemble de situations contrastées, nous nous proposons d'explorer la diversité des itinéraires professionnels des salariés d'élevage. On retient la définition d'Allaire (1991) pour qui un itinéraire professionnel agricole est "un itinéraire d'activités qui débouche sur un emploi agricole". Cette exploration s'appuie sur des entretiens réalisés auprès de salariés d'élevage de différentes régions du monde. Il ne s'agit pas de produire une connaissance ayant une validité statistique - l'échantillon ne le permet pas - mais de préciser les traits communs dans la diversité de ces itinéraires, en s'appuyant sur la confrontation de données contrastées. Nous discuterons les résultats de ce travail au regard des liens qu'ils proposent entre activité agricole et salariat, notamment à travers les circulations d'un statut professionnel à l'autre, c'est-à-dire entre celui de salarié et celui d'exploitant agricole.

\section{Méthode}

L'étude s'est appuyée sur la mobilisation des participants du projet ADD TRANS. Des enquêtes ont ainsi été réalisées dans cinq terrains (Moc Chau dans les montagnes du Nord Vietnam, Bauges dans les Alpes françaises, Roraima en Amazonie occidentale brésilienne, Paysandú dans la Pampa uruguayenne, Ferlo dans le Sahel sénégalais) sur la base d'une méthodologie commune. L'objectif initial était de disposer d'au minimum cinq enquêtes par terrain, ce qui n'a pu être atteint au Sénégal pour des raisons de disponibilité des partenaires.

Les salariés ont été rencontrés dans les fermes, connues des réseaux locaux de partenariat, dont d'autres éléments ont été analysés par ailleurs dans le projet. Nous avons toutefois été vigilants à rassembler des cas variés du point de vue tant des rythmes de présence (permanent/ régulier/saisonnier/occasionnel), que de l'âge ou du sexe. Le tableau 1 présente la situation actuelle des salariés rencontrés, sur la base de ces critères.

Pour recueillir les itinéraires des salariés, nous avons utilisé la méthodologie des récits de vie (Bertaux, 1997). Les entretiens ont ensuite été analysés selon une grille commune en trois parties :

1) l'identité et l'itinéraire du salarié : qui est-il et d'où vient-il ?

2) la description de son emploi actuel ;

3) le futur envisagé.

Les itinéraires ont, quant à eux, été construits à partir de la situation initiale du salarié et de la situation visée, des phases d'activités dont le salariat d'élevage, et des relations entre ces différents éléments.

L'analyse comparative s'est inscrite dans une approche compréhensive et a visé à identifier différents "idéaux types" d'itinéraires de salariés, qui permettent de rassembler, du fait de traits communs, différents cas et d'ordonner la diversité (Van Dam, 2005). On obtient une représentation de la réalité, mais qui peut ainsi être interrogée.

\section{Résultats}

\section{Diversité des itinéraires professionnels de salariés}

Ces itinéraires se différencient selon : i) la situation avant la phase de salariat (situation de détresse ; petite exploitation dont les revenus sont insuffisants pour vivre; combinaison d'activités rurales; jeune ayant tout juste fini ses études qui veut travailler dans l'élevage, qu'il soit fils d'exploitant ou non); ii) les objectifs visés : vivre ou non d'une exploitation et, dans le premier cas, pouvoir s'installer ou viabiliser l'existant (terres disponibles, apport financier...).

Les situations de départ identifiées renvoient à plusieurs cas de figure. On trouve tout d'abord des situations de détresse, liée à une main-d'œuvre rurale démunie, car souvent sans terre, vendant sa force de travail pour gagner un peu d'argent. D'autres ont soit une petite exploitation qui ne suffit pas pour vivre ou différentes activités non agricoles qu'ils cherchent à compléter pour raisons financières ou par goût pour l'élevage. Pour les fils d'exploitants, il arrive qu'ils se salarient un temps, faute de pouvoir ou vouloir s'installer tout de suite sur l'exploitation familiale. Enfin, certains viennent à l'élevage par passion en se salariant pour se lancer dans cette activité. Le salariat apparaît alors comme une étape transitoire du fait de l'impossibilité de s'installer comme agriculteur, dans sa région d'origine ou dans une autre, par manque de terres, de ressources financières ou par suite d'un conflit de générations entre père et fils pour la gestion de la propriété familiale. Certains salariés deviendront exploitants sur leur propre exploitation qu'ils auront achetée ou sur l'exploitation familiale. D'autres compléteront leur revenu d'exploitant par un travail salarié occasionnel hors de leur ferme. D'autres encore ne deviendront jamais exploitants indépendants car ils ne parviendront pas à réunir les conditions pour s'installer, ou finalement se satisferont du salariat. Une dernière catégorie de salariés, dont l'objectif est simplement de vendre leur force de travail pour survivre, ou pour compléter une combinaison d'activités rurales, peut arrêter dès que de meilleures opportunités se présentent ou que le projet familial évolue, comme migrer en ville. 
Tableau 1. Présentation de la situation actuelle des salariés rencontrés dans les différentes régions d'étude.

Table 1. Presentation of the current situation of the surveyed salaried employees in the different areas.

\begin{tabular}{|c|c|c|c|c|}
\hline Vietnam & France & Brésil & Uruguay & Sénégal \\
\hline $\begin{array}{l}23 \text { ans, homme, } \\
\text { salarié permanent } \\
\text { à temps plein }\end{array}$ & $\begin{array}{l}23 \text { ans, homme, salarié } \\
\text { de GE à plein-temps et travail } \\
\text { sur l'EA familiale }\end{array}$ & $\begin{array}{l}60 \text { ans, homme, } \\
\text { salarié permanent } \\
\text { à temps plein }\end{array}$ & $\begin{array}{l}29 \text { ans, homme, } \\
\text { salarié permanent } \\
\text { à temps plein, } \\
\text { petite EA à lui }\end{array}$ & $\begin{array}{l}19 \text { ans, homme, } \\
\text { salarié saisonnier }\end{array}$ \\
\hline $\begin{array}{l}28 \text { ans, homme, salarié } \\
\text { permanent à temps plein }\end{array}$ & $\begin{array}{l}46 \text { ans, homme, salarié } \\
\text { de SR à temps plein }\end{array}$ & $\begin{array}{l}29 \text { ans, homme, } \\
\text { salarié permanent } \\
\text { à temps plein }\end{array}$ & $\begin{array}{l}56 \text { ans, homme, } \\
\text { salarié permanent } \\
\text { à temps plein }\end{array}$ & $\begin{array}{l}30 \text { ans, homme, } \\
\text { salarié saisonnier }\end{array}$ \\
\hline $\begin{array}{l}29 \text { ans, femme, } \\
\text { salariée permanente } \\
\text { à temps plein }\end{array}$ & $\begin{array}{l}39 \text { ans, homme, salarié } \\
\text { d'élevage pour } 1 / 3 \text { temps, } \\
\text { travail dans une coopérative } \\
\text { pour } 2 / 3 \text { temps, et travail } \\
\text { dans l'auberge familiale }\end{array}$ & $\begin{array}{l}52 \text { ans, homme, } \\
\text { salarié permanent } \\
\text { à temps plein }\end{array}$ & $\begin{array}{l}30 \text { ans, homme, } \\
\text { journalier }\end{array}$ & $\begin{array}{l}43 \text { ans, homme, } \\
\text { salarié saisonnier, } \\
\text { petite EA à lui, } \\
\text { et intermédiaire } \\
\text { dans les marchés } \\
\text { de bétail }\end{array}$ \\
\hline $\begin{array}{l}40 \text { ans, femme, } \\
\text { salariée permanente } \\
\text { à temps plein }\end{array}$ & $\begin{array}{l}22 \text { ans, homme, salarié } \\
\text { saisonnier en élevage } \\
\text { et en station de ski }\end{array}$ & $\begin{array}{l}27 \text { ans, homme, } \\
\text { salarié permanent } \\
\text { à temps plein }\end{array}$ & $\begin{array}{l}40 \text { ans, homme, } \\
\text { salarié permanent } \\
\text { à temps plein }\end{array}$ & \\
\hline $\begin{array}{l}29 \text { ans, homme, } \\
\text { salarié permanent } \\
\text { à temps plein } \\
\text { et une petite EA à lui }\end{array}$ & $\begin{array}{l}25 \text { ans, homme, } \\
\text { salarié à temps plein }\end{array}$ & $\begin{array}{l}41 \text { ans, homme, } \\
\text { salarié permanent } \\
\text { à temps plein }\end{array}$ & $\begin{array}{l}35 \text { ans, femme, } \\
\text { salariée d'élevage } \\
\text { à temps partiel } \\
\text { et cuisinière }\end{array}$ & \\
\hline $\begin{array}{l}51 \text { ans, femme, } \\
\text { salariée permanente } \\
\text { à temps plein }\end{array}$ & $\begin{array}{l}24 \text { ans, femme, } \\
\text { salariée à temps plein }\end{array}$ & $\begin{array}{l}55 \text { ans, homme, } \\
\text { salarié permanent } \\
\text { à temps plein }\end{array}$ & & \\
\hline $\begin{array}{l}49 \text { ans, homme, } \\
\text { salarié permanent } \\
\text { à temps plein }\end{array}$ & $\begin{array}{l}25 \text { ans, homme, } \\
\text { salarié à temps plein }\end{array}$ & $\begin{array}{l}26 \text { ans, homme, } \\
\text { salarié permanent } \\
\text { à temps plein }\end{array}$ & & \\
\hline $\begin{array}{l}45 \text { ans, femme, } \\
\text { salariée permanente } \\
\text { à temps plein }\end{array}$ & $\begin{array}{l}21 \text { ans, homme, } \\
\text { salarié de GE } \\
\text { à temps plein }\end{array}$ & $\begin{array}{l}26 \text { ans, homme, } \\
\text { salarié permanent } \\
\text { à temps plein }\end{array}$ & & \\
\hline $\begin{array}{l}47 \text { ans, femme, } \\
\text { salariée permanente } \\
\text { à temps plein }\end{array}$ & $\begin{array}{l}21 \text { ans, homme, salarié } \\
\text { de SR à temps plein }\end{array}$ & $\begin{array}{l}55 \text { ans, homme, } \\
\text { salarié à temps plein }\end{array}$ & & \\
\hline $\begin{array}{l}32 \text { ans, homme, } \\
\text { salarié permanent } \\
\text { à temps plein }\end{array}$ & & $\begin{array}{l}35 \text { ans, femme, } \\
\text { salariée permanente } \\
\text { à temps partiel } \\
\text { sur l'EA et ménagère } \\
\text { sur le ranch }\end{array}$ & & \\
\hline
\end{tabular}

35 ans, homme,

salarié permanent

à temps partiel,

petite EA à lui

GE : groupement d'employeurs ; SR : service de remplacement ; EA : exploitation agricole.

\section{Cinq types d'itinéraires professionnels de salariés}

La confrontation des cas de l'ensemble des régions nous a conduits à distinguer cinq types d'itinéraires professionnels de salarié. D'une part, ces itinéraires sont qualifiés dans leur rapport au statut de chef d'exploitation (nous employons alors le terme " exploitant ") dans laquelle le salariat apparaît soit comme une situation transitoire vers le métier d'exploitant à temps plein et sur sa propre exploitation, soit en substitution au statut d'exploitant. D'autre part, ils sont reliés à l'emploi hors agriculture.

Pour les "devenir-exploitants ", le salariat agricole est un tremplin pour s'installer 
comme agriculteurs sur leur propre exploitation, et être intégrés dans une communauté professionnelle d'exploitants. Il est nettement appréhendé comme une étape dans un projet professionnel visant l'installation. Ces salariés sont soit des fils d'exploitants qui se salarient un temps, soit des travailleurs qui ont choisi ce métier par passion pour commencer leur carrière dans l'élevage. Petit à petit, certains peuvent commencer à constituer leur propre cheptel et à travailler sur leur exploitation ou aider sur l'exploitation qu'ils comptent reprendre, tout en restant salariés.

Les "compléments-exploitants" concernent des petits éleveurs dont l'exploitation ne suffit pas à couvrir leurs besoins de revenu. Ils se salarient donc selon un rythme saisonnier, ou toute l'année (temps plein ou partiel) pour compléter ce revenu jusqu'au jour où leur exploitation leur permettra de subvenir complètement à leurs besoins.

Les "substituts-exploitants" concernent ceux qui veulent travailler dans l'élevage mais sans vouloir/pouvoir assumer les responsabilités d'une exploitation indépendante. Certains trouvent dans le salariat permanent une forme de stabilité permettant l'éducation des enfants et n'ont plus envie de franchir le pas pour être exploitants indépendants ou alors seulement après la retraite. Ils se satisfont de leur statut de non-propriétaires et des avantages que leur procure leur situation (salaire mensuel fixe, congés, droits à la retraite en France ou au Brésil et à la santé). Dans d'autres cas, le salariat agricole est un moyen pour vivre pleinement leur attachement au métier d'éleveur sans subir les responsabilités associées au statut de chef d'exploitation.

Pour les "compléments-ruraux ", le salariat agricole est uniquement une des activités agricoles et non agricoles (restauration, travail en station touristique, coopérative...) possibles leur permettant de se procurer un revenu et d'assurer ainsi leur intégration sociale, sans avoir le projet de devenir exploitants indépendants.

"Les fautes de mieux " correspondent aux salariés qui souvent n'ont ni formation, ni terres. Il leur est difficile de trouver un autre emploi permettant d'assurer leur survie économique et celle de leur famille.

\section{Liens aux contextes territoriaux}

Cette étude exploratoire ne permet pas de mesurer la fréquence de chaque itinéraire dans les différentes régions d'étude, ni d'évaluer les facteurs explicatifs d'ordre territorial. Toutefois, nous pouvons signaler : i) qu'il n'y a pas de type de salariat spécifique d'une région particulière; ii) que des formes de liens entre les types d'itinéraires et les contextes territoriaux semblent se dégager.

En effet, dans tous les cas rencontrés en Amazonie et au Sénégal, le projet initial des salariés était de devenir exploitants indépendants, d'acquérir leur propriété ou de reprendre l'élevage familial. Certains projets mettent parfois du temps à aboutir et le salarié passe alors par des phases de "devenir-exploitant ", " complément-exploitant " ou "substitutexploitant". Par exemple, il peut s'agir de familles ayant de jeunes enfants et arrivant en Amazonie sans capital pour, à terme, acheter un lot de terres déjà valorisées (cultures et pâturages implantés). On retrouve également cette configuration quand un ranch embauche les familles de petites exploitations voisines pour les travaux saisonniers. Le salarié réalisant que ce statut est plutôt appréciable, finit parfois par abandonner le projet d'exploiter à son compte, et reste employé jusqu'à la fin de sa carrière, vivant ainsi son attachement à l'élevage. D'autres ne parviendront pas à rassembler les moyens pour devenir exploitants indépendants. Les perspectives d'avenir des salariés en dehors de l'élevage et même de l'agriculture se retrouvent chez des salariés d'élevage vietnamiens qui font cela "faute de mieux" et s'en iront à la première occasion (mais qui n'est ni fréquente, ni facile). Elles se retrouvent également chez des salariés français, passionnés d'élevage qui font cela un temps mais envisagent d'arrêter cette activité pour mener pleinement leurs autres activités rurales (question de temps et/ou de revenu). Ces différents itinéraires professionnels interrogent l'histoire de l'élevage de chacune des régions et l'attachement des populations aux activités d'élevage et au territoire.

\section{Discussion}

\section{Diversité des itinéraires professionnels suivis par les salariés d'élevage}

Sur l'ensemble des terrains, il ne ressort pas véritablement de salariat agricole de métier. Les itinéraires sont constitués de nombreuses expériences de salariat soit en élevage dans différentes exploitations, soit plus largement en agriculture (emploi en horticulture, arboriculture, marâ̂chage...), et parfois hors de l'agriculture. Les cinq types d'itinéraires que nous avons distingués se retrouvent en partie dans d'autres travaux (Allaire, 1991), s'intéressant aux itinéraires des travailleurs de l'agriculture (homme, femme, exploitant, aide familial, salarié). Mais du fait des terrains support de l'étude, d'autres types apparaissent dans notre travail, mettant l'accent sur les activités non agricoles, dans le parcours comme dans le futur envisagé. Nous avons également adopté une vision dynamique, non réduite au bilan de l'itinéraire passé, et prenant en compte le devenir envisagé ou en cours de mise en œuvre. La prise en compte des dynamiques professionnelles permet de qualifier plus finement les situations de travail, même si le futur envisagé n'est pas toujours celui qui se réalisera. Ainsi, dans notre cas, un individu qui n'aurait fait que du salariat n'est pas envisagé forcément comme "salarié permanent " mais comme "devenir-exploitant" s'il prépare son installation. Cette étude exploratoire du salariat en élevage offre un premier aperçu de types d'itinéraires de salariés d'élevage, qui reste à confronter et enrichir avec d'autres cas.

\section{Liens entre activité agricole et salariat}

Analyser les itinéraires de salariés nous a conduits à envisager les évolutions de statut professionnel au sein du secteur agricole, c'est-à-dire entre salarié et exploitant. De telles formes de mobilités professionnelles en agriculture ont fait l'objet de travaux d'historiens et de démographes (Lasslet et Wall, 1972; Farcy, 2004 ; Viazzo et al., 2005), qui nous amènent à formuler l'hypothèse selon laquelle ces dernières sont au cœur de la reproduction des activités agricoles. Nous sommes amenés à voir les itinéraires des salariés non pas comme de simples parcours de formation, répondant à un besoin de main-d'œuvre exprimé par les exploitants, mais comme des parcours permettant d'assurer la reproduction du groupe socioprofessionnel (Rémy, 1998). Observer un changement d'échelle, de l'individu à l'exploitation, puis au territoire permet d'envisager deux fonctions au salariat agricole. La pre- 
mière, au niveau des exploitations, est de fournir la main-d'œuvre nécessaire pour assurer la production agricole, et par là même la reproduction des exploitations, tandis que la seconde concerne les salariés en leur permettant de constituer ou reconstituer une exploitation et en tout cas de s'assurer un revenu et une intégration sociale. Autrement dit, en fonction des revenus disponibles et des possibilités d'installation, le salariat permet, d'un côté aux jeunes de parfaire leur apprentissage et d'accroître leurs ressources financières en attendant de pouvoir ou de vouloir s'installer, et de l'autre, aux exploitants en place, de faire face aux tâches et d'assurer le maintien de leurs exploitations, surtout avec l'agrandissement des structures de production ou le désengagement de la main-d'œuvre familiale.

Les facteurs limitant le " devenir " ou " rester " exploitant varient selon les terrains. Ainsi, en Amazonie, le salariat apparaît comme un mode de régulation des crises foncières. La nécessité de réduire la pression sur le foncier dans les régions du Brésil déjà largement converties à l'agriculture, a été une des raisons avancées par les pouvoirs publics brésiliens pour justifier la colonisation de l'Amazonie. La politique brésilienne de colonisation et d'intégration nationale a orienté les flux migratoires vers les fronts pionniers agricoles de l'Ouest et du Nord pour en exploiter les richesses du sol et du soussol, selon le leitmotiv: "une terre sans homme pour des hommes sans terres". Au début du XxI ${ }^{\mathrm{e}}$ siècle, soit trois ou quatre décennies après le début de la colonisation, on constate que les phénomènes migratoires se poursuivent au sein de l'espace colonisé, toujours en grande partie motivés par l'accès au foncier agricole. Le salariat, en particulier en élevage, apparaît comme une opportunité pour des jeunes ne disposant pas, ou d'assez, de terre. Le Borgne-David (1998) y voit une nouvelle stratégie de reproduction familiale ne passant plus par la conquête de terres libres et fertiles mais prenant appui sur le salariat.

On retrouve cette situation en France, mais contrairement au Brésil, l'accès au foncier s'y pense dans un contexte de déprise agricole et de concentration des exploitations. Le salariat permet d'attendre que des terres ou une exploitation se libèrent ou de trouver un exploitant déjà en place avec qui s'associer (Madelrieux et al., 2009). Au Sénégal, le salariat permet de constituer ou reconstituer un troupeau, le facteur limitant étant alors l'acquisition et l'entretien du cheptel. Ce détour professionnel par le salariat a permis par exemple, à certaines familles d'éleveurs de reconstituer tout un troupeau décimé par les sécheresses, pour ensuite vivre uniquement de leur élevage. Au Vietnam, en revanche, du fait sans doute de la pression sur le foncier encore plus forte (pour la province de Son La, incluant le district de Moc Chau, la mise en valeur des terres agricoles est passée de 68800 hectares de terres cultivées en 1995 à 168500 hectares en 2007, d'après l'Office général de statistiques du Vietnam), le salariat apparaît davantage comme une voie pour limiter l'exode rural et maintenir des activités d'élevage dans les campagnes, que comme un vivier de futurs exploitants. Des salariés agricoles restent dans les régions rurales même s'ils y sont sous-employés, et leur mobilité reste très liée à l'opportunité de mieux gagner leur vie en quittant ce milieu estimé peu attractif.

\section{Conclusion}

Cette étude exploratoire montre des salariés d'élevage s'inscrivant dans des itinéraires de "devenir exploitant", de "complément exploitant", de "substitut exploitant ", de "complément rural ", de "faute de mieux ". L'évolution des statuts, de salarié à exploitant, permet de gérer : i) une main-d'œuvre rurale; ii) des possibilités territoriales parfois restreintes d'installation en tant qu'exploitant indépendant, du fait des difficultés à réunir les ressources nécessaires (foncier, cheptel, équipements, ressources financières, main-d'œuvre); iii) un besoin de maind'œuvre dans des exploitations pour assurer durablement leur production. Ces itinéraires peuvent être organisés ou facilités par l'existence de réseaux professionnels, d'interconnaissances, de proximité, ou familiaux. Il reste cependant à préciser l'importance de chaque type d'itinéraire sur chacun des terrains, et à analyser ces rapports à la mobilité en tenant compte à la fois de l'histoire et de la culture agraire locale mais aussi des politiques publiques (sectorielles et territoriales) des États concernés.

\section{Références}

Allaire G. Itinéraires et identités professionnelles des travailleurs de I'agriculture. Economie et Sociologie Rurales 1991 ; 3 : 175-211.
Bertaux D. Les récits de vie. Perspective ethnosociologique. Paris : Nathan, 1997.

CEDAG. Groupements d'employeurs: comprendre pour mieux accompagner. Rennes: Doc CEDAG, 2001

Chabanet G, Dedieu B, Servière G, Tchakérian $E$, Lemery $B$. Le salariat partagé en région d'élevage. Caractéristiques et fonctionnement des groupements d'employeurs en Auvergne et Limousin. Cah Agric $2000 ; 9$ : 23-8.

Cournut S, Dedieu B, Hostiou N, Servière G. L'organisation du travail en exploitations familiales d'élevage. Enseignements d'une analyse réalisée en France et dans quatre pays du Sud. Cah Agric 2010; 19: epub. doi : 10.1684/agr.2010.0420

Dupré L. Salariat non familial en élevage laitier de montagne: qualifications et qualités. Cah Agric 2010 ; 19 : epub. doi : 10.1684/agr.2010.0423

Farcy JC. La jeunesse rurale dans le France du XIXème siècle. Collection "vivre I'histoire". Paris : éditions Christian, 2004

Findeis $\mathrm{JL}$, Vandeman AM, Larson JM, Runyan JL. The dynamics of hired farm labour. Constraints and community responses. Wallingford (Great Britain) : CABI Publishing, 2002.

Harff $Y$, Lamarche $H$. Le travail en agriculture: nouvelles demandes, nouveaux enjeux. Economie Rurale 1998 ; 244 : 3-11.

Hostiou N, Dedieu B, Madelrieux S, Duy KP, Binh TV. Relations entre organisation du travail et taille des exploitations laitières: une étude à Moc Chau (Vietnam). Cah Agric 2010; 19: epub. doi : 10.1684/agr.2010.0404.

Hostiou N, Dedieu B. Diversity of forage system work and adoption of intensive techniques in dairy cattle farms of Amazonia. Agron Sustain Dev 2009 ; 29 : 535-44.

ILO (International Labour Organization). International Labour Conference, 97th Session, Report IV. Geneva: International Labour Office, 2008. www.ilo.org/wcmsp5/groups/public/-ed norm/-relconf/documents/meetingdocument/ wcms_091721.pdf\#page $=33$

Lasslet P, Wall R. Household and family in past time. Cambridge: Cambridge University Press, 1972.

Le Borgne-David A. Les migrations paysannes du sud-Brésil vers l'Amazonie: "le salariat plutôt que la malaria ». Paris: L'Harmattan, 1998.

Madelrieux S, Dupré L, Rémy J. Itinéraires croisés et relations entre éleveurs et salariés dans les Alpes du Nord. Economie Rurale 2009 ; (313314) : 6-21.

Neiman G. Trabajo de campo. Produccion, technologia y empleo en el medio rural. Buenos Aires: ediciones CICCUS, 2001.

Rémy J. Désastre ou couronnement d'une vie? La vente aux enchères à la ferme. Ruralia 1998; $3: 67-90$.

Van Dam D. Les agriculteurs bio, vocation ou intérêt? Namur: Presses universitaires de Namur, 2005.

Viazzo PP, Aime M, Allovio S. Crossing the boundary: Peasants, shepherds, and servants in a western Alpine community. The History of the Family 2005 ; 10 : 387-405.

Wane A, Ancey V, Diao-Camara A, Kâ S. L'économie pastorale face aux incertitudes - Le salariat au Ferlo (Sahel sénégalais). Cah Agric 2010; 19 : epub. doi : 10.1684/agr.2010.0427 